\title{
GESTÃO DO CONHECIMENTO EXPLÍCITO NAS ORGANIZAÇÕES QUE NÃO POSSUEM BASE DE DADOS ELETRÔNICA
}

\section{EXPLICIT KNOWLEDGE MANAGEMENT IN ORGANIZATIONS THAT DO NOT HAVE ELECTRONIC DATABASE}

\author{
Data do recebimento do artigo: 14/10/2013 \\ Data do aceite do artigo: 16/9/2014 \\ Data da publicação: 8/12/2014 \\ Processo de Avaliação: Double Blind Review
}

\author{
Maria Lúcia Gili Massi ${ }^{1}$ \\ Doutora em Letras Clássicas pela Universidade de São Paulo \\ FIPEN-Faculdade Instituto Paulista de Ensino
}

\section{RESUMO}

Na sociedade da informação, o conhecimento é o principal fator de produção. Para se manter competitivas, as organizações são impelidas pelo mercado global a fazerem a gestão do conhecimento. A tecnologia da informação tem se mostrado grande aliada no processo de estruturar, armazenar e disseminar o conhecimento para toda a empresa. No entanto, há organizações que não dispõem de base de dados eletrônica e outras instituições que não podem utilizar as ferramentas eletrônicas pela impossibilidade de correrem riscos de divulgação de informações de segurança nacional. Tal dificuldade estimulou a realização deste estudo que objetiva verificar se a gestão do conhecimento explícito nas organizações que não possuem base de dados eletrônica pode ser feita pelo treinamento. A pesquisa bibliográfica revelou que o treinamento tem sido utilizado como ferramenta para a disseminação do conhecimento explícito.

Palavras Chave: Treinamento. Gestão do conhecimento sem base de dados eletrônica. Gestão do conhecimento. Conhecimento explícito.

\begin{abstract}
In the information society, knowledge is the main factor of production. To stay competitive, organizations are driven by the global market to make knowledge management. Information technology has shown great ally in the process of structuring, storing and disseminating knowledge across the enterprise. However, there are organizations that do not have electronic databases and other institutions that may not use electronic tools for the inability to take risks disclosure of national security. This difficulty prompted this study which aims to determine whether the management of explicit knowledge in organizations that do not have electronic database can be done by training. A literature search revealed that the training has been used as a tool for the dissemination of explicit knowledge.
\end{abstract}

\footnotetext{
${ }^{1}$ Autor para correspondência: Autor para correspondência: Faculdade Instituto Paulista de Ensino - Rua Euclides da Cunha, 377, Centro, Osasco, SP, CEP 06016-030. mlgmassi @ fipen.edu.br.
} 
Key-Words: training, knowledge management without electronic database, knowledge management, knowledge explicit. 


\section{INTRODUÇÃO}

Até o século XIX o desenvolvimento do conhecimento nas organizações era muito lento. As bases do meio de produção eram a manipulação dos recursos naturais e a utilização da força física. A partir do século XIX, o progresso tecnológico e o desenvolvimento das ciências aceleraram o processo de geração de conhecimento pelo uso mais acentuado da informação nos processos operacionais (Inazawa, 2009).

Com o aprimoramento da capacidade produtiva humana, dos meios de comunicação, das técnicas de produção e transmissão do conhecimento e da ciência, elevando o conhecimento ao nível de principal fator da produção no século XXI, surge a economia do conhecimento (Inazawa, 2009).

Atualmente, as organizações sabem que o que gera os recursos necessários para se alcançar a riqueza não são mais os recursos naturais e a força física, ou mesmo ferramentas mecânicas e fábricas, e sim ativos baseados em conhecimentos. Por isso, o trabalho exige maior capacidade de cognição por parte dos trabalhadores, maior nível de aprendizagem e gestão do conhecimento gerado. $\mathrm{Na}$ era da informação e do conhecimento em que vivemos, é exigido que os trabalhadores sejam criativos, tenham ideias e inovem, pois o trabalho físico é realizado pelas máquinas. O problema hoje é como gerir esse conhecimento, para que a empresa tenha vantagem competitiva (Inazawa, 2009).

São inúmeras as ferramentas utilizadas pelas organizações para fazerem a disseminação do conhecimento. As empresas de grande porte utilizam a tecnologia da informação (Carvalho et al., 2006). No entanto, há empresas de menor porte que não dispõem de base de dados eletrônica e há outras instituições que, embora sejam grandes, não podem utilizar as ferramentas eletrônicas pela impossibilidade de correrem riscos de divulgação de informações de segurança nacional (Carvalho et al.,2006). Diante dessa situação e tendo em vista a importância que a gestão do conhecimento tem para a competitividade das empresas, este estudo visa verificar se a gestão do conhecimento explícito nas organizações que não possuem base de dados eletrônica pode ser feita pelo treinamento.

\section{MATERIAIS E MÉTODOS}

O estudo será baseado em pesquisa bibliográfica feita em trabalhos acadêmicos sobre gestão do conhecimento nas organizações.

\section{RESULTADOS E DISCUSSÃO}

A informação e o conhecimento são as principais armas competitivas da nossa era (Fialho et al., 2006); um insumo fundamental ao processo produtivo, que fortalece o processo de globalização das economias e dos mercados, migrando da gestão 
preconizada dos modelos da sociedade industrial para os modelos da sociedade da informação (Carvalho et al., 2006). Por sua relevância para a sobrevivência das empresas, o conhecimento tem se destacado como um bem econômico precioso (Ceci, 2010) e, por isso, precisa ser gerido para evitar que ele se perca ao longo do tempo (Moresi, 2001).

A gestão do conhecimento, sendo um processo de mudança cultural (Igarashi et al., 2008), pode ser definida como um conjunto de atividades que busca desenvolver e controlar todo tipo de conhecimento em uma organização, visando a sua utilização na consecução dos objetivos (Moresi, 2001). Trata-se de um processo que cria vantagem competitiva sustentável para a empresa porque surge das pessoas que se relacionam no ambiente de trabalho, promovendo a aprendizagem da equipe. Tal aprendizagem desenvolve a flexibilidade nas pessoas para fazerem, sistematicamente, o realinhamento da empresa diante das incertezas do cenário (Quinn et al., 1997; Thomke \& Reinertsen, 1998, apud Silva, 2004; Igarashi et al., 2008).

A principal teoria da criação e disseminação do conhecimento nas organizações surgiu com os estudos de Nonaka e Takeuchi, 1997. Para esses pesquisadores, o conhecimento organizacional baseia-se na combinação de dois tipos de conhecimento humano: o tácito e o explícito. Os conhecimentos tácitos referem-se às experiências dos indivíduos, suas habilidades, seu know-how, seus valores e outros elementos intangíveis. $\mathrm{O}$ conhecimento explícito diz respeito ao conhecimento codificado e explicitado em algum suporte, como em um manual de procedimentos de operação de uma máquina por exemplo.

Para os autores da teoria, o conhecimento organizacional tem origem na contínua conversão do conhecimento tácito em explícito e explícito em tácito conforme o esquema seguinte:

1. Socialização: conversão de conhecimento tácito em conhecimento tácito.

Exemplo: Uma pessoa conversando transfere para outra pessoa seus conhecimentos sobre determinada coisa.

2. Externalização: conversão de conhecimento tácito em explícito.

Exemplo: Realização de uma prova na faculdade.

3. Combinação: conversão de conhecimento explícito em explícito.

Exemplo: Com base no banco de dados dos alunos de uma faculdade, a Secretaria emite a lista de presença dos alunos.

4. Internalização: conversão de conhecimento explícito em tácito.

Exemplo: Conhecimento adquirido pelo aluno quando lê um livro.

Os conhecimentos que surgem nas empresas são resultantes de uma interação contínua e dinâmica entre os tipos tácitos e explícitos. O conhecimento explícito é 
aquele formalmente encapsulado e compartilhado entre os empregados, visando a sua utilização na realização dos trabalhos demandados (Moresi, 2001).

Desse modo, o conhecimento organizacional não existe por si só, ele depende da soma do conhecimento de cada empregado, que precisa ser compartilhado entre os membros da organização, e, para que ele flua, além de dimensões intangíveis, como um ambiente propício, uma cultura de aprendizagem, uma liderança apoiadora e outros (Odelius et al., 2011), é preciso que a organização tenha mecanismos para coletar, estruturar e disseminar esse conhecimento (Moresi, 2001).

A disseminação do conhecimento, compreendida como a transformação do conhecimento em saber incorporado pelas pessoas, é condição prévia para transformar experiências isoladas em informação que toda a empresa possa utilizar. Assim, só pode haver absorção de conhecimento pelo empregado quando existe a disseminação (Carvalho et al., 2006).

A difusão do conhecimento consiste na prática de transferência do conhecimento entre as pessoas, que pode ser pela feita mediante ações informais (Camillis \& Antonello, 2010), como treinamento no trabalho, chamado de on the job (Popadiuk \& Santos, 2010), conversas nos corredores da empresa e telefônicas, técnicas de brainstorming e outros meios pelos quais uma pessoa externaliza seu conhecimento tácito em conceitos explícitos, e ações estruturadas que permitem a mobilidade do conhecimento pela empresa toda (Carvalho et al., 2006).

Muitos estudos mostram a dificuldade que as empresas têm de socializar e externalizar os conhecimentos (Sabbadini \& Ferreira Filho, 2006).

Em relação à utilização de práticas informais como ferramenta para divulgação de conhecimento, há pesquisas empíricas mostrando que, apesar de as empresas afirmarem que incentivam e facilitam o compartilhamento do conhecimento entre seus membros, $80 \%$ deles, ao serem consultados, responderam que não existe um incentivo para a documentação do conhecimento da empresa, sistemática que possibilitaria a gestão do conhecimento explícito. O motivo que dificulta a obtenção de informações, segundo $73 \%$ dos respondentes, é a falta de sistematização para a coleta, organização e divulgação da informação (Lima, 2004).

Quando a estratégia de gestão de conhecimento da empresa está voltada para o conhecimento explícito, o foco é na armazenagem do conhecimento em uma base de dados, para obter ser acessado e utilizado por todos na organização. Essa estratégia se concentra principalmente em tecnologia da informação (TI). Nesse sentido, a TI tem sido muito referida pelos especialistas como uma ferramenta formal altamente praticada para a propagação do conhecimento explícito, cabendo-lhe alavancar, conduzir e direcionar a gestão do conhecimento no interior das empresas (Igarashi et al., 2008). As empresas de grande porte utilizam e-mails; internet, intranet, chats, mensagens instantâneas como estratégias para a disseminação dos conhecimentos (Carvalho et al., 2006).

No entanto, nem todas as organizações podem utilizar meios eletrônicos para fazer a disseminação do conhecimento. Para algumas instituições, a informação e o 
conhecimento são dados confidenciais e a sua disseminação inapropriada pode trazer grandes prejuízos (Carvalho et al., 2006). Outras empresas não dispõem de meios de disseminação da informação respaldadas por base eletrônica, ou quando possuem tais meios, não possuem recursos financeiros para investir em sistemas de bancos de dados que sustentem as informações a serem propagadas.

Instituições em que a informação e o conhecimento são bens estratégicos, como o caso da NASA-KSC nos USA, no final da década de 1980, e dos Centros de Pesquisa brasileiros, enquadrados como de Defesa Nacional, a disseminação do conhecimento é feita com pessoas capazes de transferir o conhecimento de indivíduo para indivíduo nutrindo um processo natural de mentoring e treinamento, ou mediante um processo de educação formal (Carvalho et al., 2006). Atualmente, a NASA dispõe de um Portal do Conhecimento aberto à participação pública mundial (Sacramento, 2011).

As instruções de trabalho e as normas de procedimentos encapsulados em documentos formais das empresas são também fontes de disseminação do conhecimento explícito muito utilizadas (Carvalho et al., 2006), que independem de meios eletrônicos, mas são de alcance restrito.

Outras práticas para a disseminação do conhecimento explícito, que não dependem de bases de dados eletrônicas, são as defesas de tese, apresentação de trabalhos de conclusão de curso, de mestrado, doutorado, participação em congressos e seminários, anais de eventos científicos, livros, publicações técnicas, treinamentos formais e informais (Carvalho et al., 2006).

O treinamento a distância pode ser feito online ou pelo sistema de correspondência como faz o Instituto Universal Brasileiro (IUB), que transfere o conhecimento codificado, como regras e procedimentos, mediante uma apostila. Seguindo esse método do IUB de gestão do conhecimento explícito, os empregados poderiam ser incentivados pela unidade de treinamento da empresa a lerem apostilas ou manuais e a realizam provas para medir o conhecimento explícito internalizado (Joia, 2007, apud Lemos \& Jóia, 2012).

A gestão do conhecimento explícito, além do treinamento on the job, também pode ser feita por meio de programas sistematizados e formais de Treinamento \& Desenvolvimento. Esse enfoque se refere a uma ação de aprendizagem totalmente planejada, dirigida e pautada por princípios instrucionais, ao contrário da maneira natural ou não planejada do treinamento no trabalho, considerada mais emergencial e espontânea (Coelho Júnior \& Borges-Andrade, 2008).

Há estudo mostrando que o treinamento formal é uma ferramenta muito utilizada pelas empresas para disseminar conhecimentos. No entanto, os participantes desse estudo, ao serem indagados sobre a validade dessa ferramenta para a propagação do conhecimento explícito, responderam que nem sempre os treinamentos formais alcançam todos os empregados e, muitas vezes, deixam de fora aqueles que realmente necessitam da informação (Carvalho et al, 2006).

Por outro lado, a pesquisa feita por Benossi, 2009, revelou que as organizações, pressionadas pelo mercado, estão fazendo a inter-relação entre gestão do conhecimento 
e a área de Treinamento e Desenvolvimento de Pessoas (T\&D). A pesquisadora concluiu seu trabalho afirmando que há convergência entre os processos e T\&D e Gestão do Conhecimento, visto que o crescimento e desenvolvimento corporativo pressupõem a capacidade da empresa em criar conhecimento novo, compartilhando-o entre os empregados e agregando-o, de forma concreta, através do aprendizado, a processos, serviços e produtos.

Ainda que o treinamento formal, muitas vezes, não alcance todos os empregados que necessitam da informação para agregar novos valores ao trabalho, conforme demonstrou a pesquisa mencionada, o treinamento, interno ou externo, on the job ou formal, tem se revelado como uma importante ferramenta para a disseminação do conhecimento explícito (Benossi, 2009). Entretanto, nenhum instrumental, para transferência de conhecimento, será eficaz se não contar com uma cultura organizacional que apóie a disseminação e a internalização das informações pelos empregados, e a decorrente aplicação no trabalho, ocasião em que a ação de treinamento se efetiva pela mudança de comportamento promovida.

Qualquer instrumento pode ser um grande aliado na gestão do conhecimento, desde que ele esteja adaptado ao contexto organizacional.

Diante das evidências reveladas pela pesquisa bibliográfica, pode-se concluir que o objetivo que norteou este estudo foi atingido, pois foi verificado que a gestão do conhecimento explícito nas organizações que não possuem base de dados eletrônica pode ser feita pelo treinamento.

\section{REFERÊNCIAS}

BENOSSI, G.. Gestão do conhecimento no treinamento e desenvolvimento de Pessoas. Dissertação de mestrado em Ciência da Informação, Pontifícia Universidade Católica, Campinas, São Paulo. 2009.

CAMILLIS, P. K. de \& ANTONELLO, C. S.. Um estudo sobre os processos de aprendizagem dos trabalhadores que não exercem função gerencial. RAM 11: 4-42. 2010.

CARVAlHO, A. R. de S.; MASCARENHAS, C. C. de \& OllVEIRA, E. A. de A. Ferramentas de disseminação do conhecimento em uma instituição de C\&T de defesa nacional. Revista de Gestão da Tecnologia e Sistemas de Informação 3: 77-92. 2006.

CECI, F. Um modelo semiautomático para a construção e manutenção de ontologias a partir de bases de documentos não estruturados. Dissertação de Mestrado, Universidade Federal de Santa Catarina, Florianópolis, SC.2010.

COELHO, F. A. Jr. \& BORGES-ANDRADE, J. E. Uso do conceito de aprendizagem em estudos relacionados ao trabalho e organizações. Paidéia 18: 221-234. Disponível em www.scielo.br/paideia. Consulta em 23.03.2013. 2008 
FIALHO, F. A. P.; MACEDO, M.; SANTOS, Neri dos \& MITIDIERI, T. da C.. Gestão do conhecimento e aprendizagem: as estratégias competitivas da sociedade pós-industrial. Florianópolis: Visual Book. 2006.

IGARASHI, W.; IGARASHI, D. C. C.: VIERIA, E. M. F. \& TODESCO, J. L. Investigação no contexto brasileiro sobre gestão do conhecimento/aprendizagem/tecnologia de informação: pesquisa realizada na Scientific Electronis Library Online. Cadernos EBAPE.BR 6:1-18. 2008.

INAZAWA, F.K. O papel da cultura organizacional e da aprendizagem para o sucesso da gestão do conhecimento. Perspectivas em Ciência da Informação, v.14, n.3, p.206220, set./dez.2009.

INSTITUTO UNIVERSAL BRASILEIRO. Disponível em www.institutouniversal.com.br. Consulta em 23.03.2013.

LEMOS, B. \& JÓIA, L. A. Fatores relevantes à transferência de conhecimento tácito em organizações: um estudo exploratório. Gest. Prod. São Carlos 19: 233-246. 2012.

LIMA, A.M. de S. Os impactos da globalização no mundo do trabalho. TERRA E CULTURA 39: 32-49. 2004.

MORESI, E. A. D. Inteligência organizacional: um referencial integrado. Ci. Inf. Brasília 30: 35-46. 2001

NONAKA, I. \& TAKEUCHI, H. Criação de conhecimento na empresa: como as empresas japonesas geram a dinâmica da inovação. Rio de Janeiro: Campus. 1997.

ODELIUS, C. C.; ABBAD, G. da S.; RESENDE, P.C.Jr.; SENA, A. de C.; VIANA, C. R.; FREITAS, T, L. \& SANTOS, T. C. N. dos. Processos de aprendizagem, competências aprendidas, funcionamento, compartilhamento e armazenagem de conhecimentos em grupos de pesquisa. CADERNOS EBAPE 9: 199-220. 2011.

POPADIUK, S. \& SANTOS, A. E. M. dos. Conhecimentos tácito, explícito e cultural no planejamento da demanda. Revista de Gestão de Tecnologia e Sistemas de Informação 7: 205-226. 2010.

SABBADINI, F. S. \& FERREIRA FILHO, E.P. Teoria da criação do conhecimento organizacional aplicada a uma indústria de bebidas. REA 5: 1-14. 2006.

SACRAMENTO, J. M. N. Ferramentas para adequação das linhas de pesquisas de instituto de pesquisa: o exemplo do IPEN. Tese de doutorado em Tecnologia Nuclear Aplicações, Instituto de Pesquisas Energéticas e Nucleares, Universidade de São Paulo, São Paulo. 2011.

SILVA, S. L. da. Gestão do conhecimento: uma revisão crítica orientada pela abordagem da criação do conhecimento. Ci. Inf., Brasília 33: 143-151. 2004. 